\title{
Categorical Tori
}

Nora GANTER

School of Mathematics and Statistics, The University of Melbourne, Parkville, Victoria 3010, Australia

E-mail: noraganter@gmail.com

URL: http://researchers.ms.unimelb.edu.au/〜nganter@unimelb/

Received September 23, 2017, in final form January 31, 2018; Published online February 17, 2018 https://doi.org/10.3842/SIGMA.2018.014

\begin{abstract}
We give explicit and elementary constructions of the categorical extensions of a torus by the circle and discuss an application to loop group extensions. Examples include maximal tori of simple and simply connected compact Lie groups and the tori associated to the Leech and Niemeyer lattices. We obtain the extraspecial 2-groups as the isomorphism classes of categorical fixed points under an involution action.
\end{abstract}

Key words: categorification; Lie group cohomology

2010 Mathematics Subject Classification: 22E99; 18D99

\section{Introduction}

By a categorical group, or a 2-group, we mean a monoidal groupoid $(\mathcal{G}, \bullet, 1)$ with weakly invertible objects. If $\mathcal{G}$ is a Lie groupoid, the monoidal structure is required to be locally smooth in an appropriate sense [12]. In this situation, one speaks of a Lie 2-group. Categorical groups play as important a role in string theory as groups do in particle physics [1], and a number of prominent groups seem to be most naturally understood via their categorical refinements. Most famously, the infinite-dimensional groups String(n) come from finite-dimensional Lie 2-groups [12]. Also, Weyl groups and some of the sporadic groups, including the Monster, are known or conjectured to be the isomorphism classes of categorical groups (see Section 5). In recent years, there is a growing understanding that the categorical nature of these groups is worth exploring, and we will see that this perspective sheds new light on some old and important mathematics. The purpose of this paper is to give a simple and hands-on description of a basic class of examples, namely all central extensions of Lie 2-groups of the form

$$
1 \longrightarrow \mathrm{pt} / / U(1) \longrightarrow \mathcal{T} \longrightarrow T \longrightarrow
$$

where $T$ is a compact torus, and pt $/ / U(1)$ is the one-object groupoid with Aut(pt) $=U(1)$. We will refer to such a $\mathcal{T}$ as a categorical torus. Categorical tori are important for a number of reasons: first, they turn up as maximal tori of Lie 2-groups. So, any character theory of Lie 2-groups should sensibly start here. Second, categorical tori incorporate many aspects relevant to the construction of sporadic groups (see Sections 5 and 6). Finally, the theory of Lie 2-groups is closely related to that of loop groups, a point we will explore in Section 7. Our approach is to work from 2-groups to loop groups. In particular, our first construction of $\mathcal{T}$ does not involve loops or any other infinite-dimensional objects. The transgression machine of [17] then yields a simple description of central extensions of the loop group $\mathcal{L} T$. What we hope to get across is that categorical groups can be fairly simple to construct and easy to work with and that some of

This paper is a contribution to the Special Issue on Moonshine and String Theory. The full collection is available at https://www.emis.de/journals/SIGMA/moonshine.html 
the complicated features of loop groups are merely the shadow of rather obvious phenomena on the categorical side. This is the first in a series of papers describing the representation theory of categorical tori.

\section{Constructions of categorical tori}

Let $\Lambda^{\vee}$ be a free $\mathbb{Z}$-module of finite rank, and let $J$ be an integer-valued bilinear form on $\Lambda^{\vee}$. Tensoring $\Lambda^{\vee}$ over $\mathbb{Z}$ with the short exact sequence

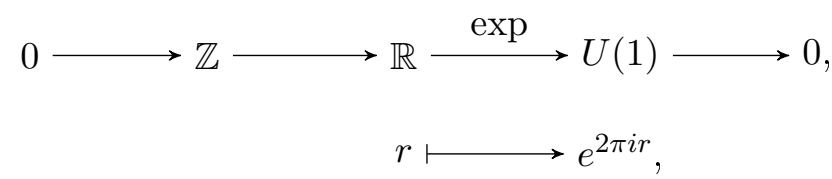

we obtain the short exact sequence

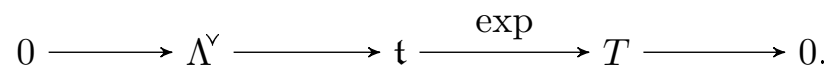

Here $T$ is the compact torus with coweight lattice $\Lambda^{\vee}$, and $\mathfrak{t}$ is its Lie-algebra. We will give three equivalent constructions of the categorical torus associated to $\left(\Lambda^{\vee}, J\right)$.

Construction 2.1. Let $\mathfrak{t}$ act on $\Lambda^{\vee} \times U(1)$ by

$$
\begin{aligned}
\mathfrak{t} \times \Lambda^{\vee} \times U(1) & \longrightarrow \Lambda^{\vee} \times U(1), \\
(x, m, z) & \longmapsto(m, z \cdot \exp (J(m, x))) .
\end{aligned}
$$

Then the strict categorical group $(\mathcal{T}, \bullet, 0)$ associated to the crossed module

$$
\begin{aligned}
\Psi: \Lambda^{\vee} \times U(1) & \longrightarrow \mathfrak{t}, \\
(m, z) & \longmapsto m
\end{aligned}
$$

is a categorical torus.

Explicitly, the Lie groupoid $\mathcal{T}$ has objects $\mathfrak{t}$ and arrows $\mathfrak{t} \ltimes\left(\Lambda^{\vee} \times U(1)\right)$, which we write as

$$
x \stackrel{z}{\longrightarrow} x+m
$$

with $x \in \mathfrak{t}, m \in \Lambda^{\vee}$ and $z \in U(1)$, to indicate source and target. Composition in $\mathcal{T}$ is

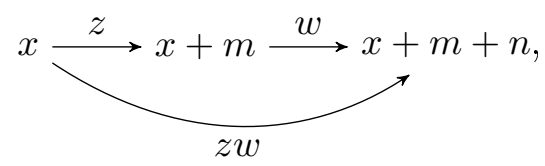

and tensor multiplication is

$$
\begin{aligned}
& x \bullet y=x+y, \\
& (x \stackrel{z}{\longrightarrow} x+m) \bullet(y \stackrel{w}{\longrightarrow} y+n)=(x+y \stackrel{z w \exp (J(m, y))}{\longrightarrow} x+y+m+n) .
\end{aligned}
$$


The unit object is 0 , and the associativity and unit isomorphisms are identities. This gives $\mathcal{T}$ the structure of a group object in the category of Lie groupoids. When we want to emphasize the dependence on $J$, we will use the notation $\bullet_{J}$ for $\bullet$. In the following, we will use the notation $X / / G$ for the translation groupoid associated to the action of a group $G$ on a space $X$. With this notation, the underlying groupoid of Construction 2.1 is

$$
\mathcal{T} \cong\left(\mathfrak{t} / / \Lambda^{\vee}\right) \times(\mathrm{pt} / / U(1)),
$$

where $\Lambda^{\vee}$ acts on $\mathfrak{t}$ by translations.

Construction 2.2. Consider the torus $T$ as a Lie groupoid with only identity arrows, and let

$$
p: \mathfrak{t} / / \Lambda^{\vee} \stackrel{\sim}{\longrightarrow} T
$$

be the equivalence of Lie-groupoids sending the object $x$ to $\exp (x)$. Note that $p$ does not possess a continuous inverse. The language developed in [12] interprets

$$
\mathfrak{T}=T \times \mathrm{pt} / / U(1),
$$

together with the data inherited from Construction 2.1, as a 2-group object with multiplication

$$
\mathfrak{T} \times \mathfrak{T} \stackrel{\sim}{\sim} \mathcal{T} \times \mathcal{T} \stackrel{\bullet}{\longrightarrow} \stackrel{\sim}{\longrightarrow}
$$

in a suitable localization of the bicategory of Lie-groupoids. The equivalences in (2.1) are those induced by $p$. Different communities have different language for the 1-morphisms in this localized bicategory Bibun. Depending on taste, the reader may wish to think of this multiplication on $\mathfrak{T}$ as a zig-zag, a span, an orbifold map, an anafunctor, or a bibundle.

Our third construction is as a multiplicative bundle gerbe in the sense of [3] and [5].

Construction 2.3. Let $\mathbf{L}$ be the line bundle over $T \times T$ with multipliers

$$
\begin{aligned}
f_{(m, n)}: \mathfrak{t} \times \mathfrak{t} & \longrightarrow U(1), \\
(x, y) & \longmapsto \exp (J(m, y)),
\end{aligned}
$$

$(m, n) \in \Lambda^{\vee} \times \Lambda^{\vee}$. We claim that over $T \times T \times T$ we have a canonical isomorphism

$$
\alpha: \mathrm{m}_{12}^{*} \mathbf{L} \otimes \operatorname{pr}_{12}^{*} \mathbf{L} \cong \mathrm{m}_{23}^{*} \mathbf{L} \otimes \operatorname{pr}_{23}^{*} \mathbf{L},
$$

where $\mathrm{pr}_{i j}$ is the projection onto factors $i$ and $j$ and $\mathrm{m}_{i j}$ is given by multiplication of these two factors. Indeed, source and target of $\alpha$ have identical multipliers

$$
\begin{aligned}
f_{(k, m, n)}: \mathfrak{t} \times \mathfrak{t} \times \mathfrak{t} & \longrightarrow U(1), \\
(x, y, z) & \longmapsto \exp (J(k, y)+J(k, z)+J(m, z)),
\end{aligned}
$$

$(k, m, n) \in \Lambda^{\vee} \times \Lambda^{\vee} \times \Lambda^{\vee}$. The pair $(\mathbf{L}, \alpha)$ equips the trivial bundle gerbe over $T$ with a multiplicative structure. It is well known (e.g., [16, Theorem 3.2.5]) that the data of a multiplicative bundle gerbe over $T$ are equivalent to those of a Lie 2 -group extension of $T$ by pt //U(1).

Explicitly, the line bundle $\mathbf{L}$ is constructed as

$$
\mathbf{L}=\mathbb{C} \times \mathfrak{t} \times \mathfrak{t} / \sim
$$

with

$$
(z, x, y) \sim(z \cdot \exp (J(m, y)), x+m, y+n)
$$


for $(m, n) \in \Lambda^{\vee} \times \Lambda^{\vee}$. The trivial bundle gerbe over $T$ corresponds to the groupoid $\mathcal{I}$ whose objects are pairs $(t, L)$ with $t \in T$ and $L$ a hermitian line, and whose arrows

$$
(t, L) \longrightarrow\left(t, L^{\prime}\right)
$$

are the unitary isomorphisms from $L$ to $L^{\prime}$. The line bundle $\mathbf{L}$ equips $\mathcal{I}$ with the monoidal structure

$$
\left(s, L_{1}\right) \circledast\left(t, L_{2}\right)=\left(s \cdot t, \mathbf{L}_{s, t} \otimes L_{1} \otimes L_{2}\right),
$$

whose associativity isomorphisms

$$
\alpha_{r, s, t}: \mathbf{L}_{r \cdot s, t} \otimes \mathbf{L}_{r, s} \cong \mathbf{L}_{r, s \cdot t} \otimes \mathbf{L}_{s, t}
$$

are encoded in $\alpha$.

Proposition 2.4. The three constructions yield equivalent extensions of $T$ by $\mathrm{pt} / / U(1)$.

Proof. It is clear that Construction 2.1 and Construction 2.2 are equivalent. To see their equivalence with Construction 2.3, let $F$ be the functor

$$
\begin{aligned}
F: \mathcal{T} & \longrightarrow \mathcal{I} & & \\
x & \longmapsto(\exp (x), \mathbb{C}) & & \text { on objects }, \\
(x, m, z) & \longmapsto z & & \text { on arrows. }
\end{aligned}
$$

We want to make $F$ into a monoidal equivalence. For this, we need to specify an isomorphism

$$
\varepsilon:(1, \mathbb{C}) \cong F(0)
$$

and a natural isomorphism

$$
\phi: F(-) \circledast F(-) \Longrightarrow F(-\bullet-),
$$

satisfying the usual unit and associativity conditions. Take $\varepsilon=i d$ and note that $\phi$ needs to assign an isomorphism

$$
\phi_{x, y}: \mathbf{L}_{\exp (x), \exp (y)} \longrightarrow \mathbb{C}
$$

to each pair of objects $(x, y)$ of $\mathcal{T}$. By construction of $\mathbf{L}$, we have a trivialization of $\mathbf{L}$ over $\mathfrak{t} \times \mathfrak{t}$, and this trivialization serves as our $\phi$. One checks that $(F, \phi, \varepsilon)$ satisfies the axioms of a monoidal equivalence from $(\mathcal{T}, \bullet, 0)$ to $(\mathcal{I}, \circledast,(1, \mathbb{C}))$.

Remark 2.5. Construction 2.3 takes the sum of two bilinear forms to the tensor product of the corresponding multiplicative bundle gerbes.

Definition 2.6. We will write $J^{t}$ for the bilinear form

$$
J^{t}(m, n)=J(n, m) .
$$

We say that $J$ is symmetric if $J=J^{t}$ and that $J$ is skew symmetric if $J=-J^{t}$. A symmetric bilinear form $I$ is called even if

$$
I(m, m) \in 2 \mathbb{Z} \quad \text { for } m \in \Lambda^{\vee} .
$$

Proposition 2.7. The Lie 2-groups $\left(\mathcal{T}, \bullet_{J}, 0\right)$ and $\left(\mathcal{T}, \bullet_{J}^{t}, 0\right)$ are equivalent as Lie 2-group extensions of $T$ by $\mathrm{pt} / / U(1)$. 
Proof. A monoidal equivalence is given by the functor

$$
\begin{array}{rlrl}
F: \mathcal{T} & \longrightarrow \mathcal{T}, & \\
\mathfrak{t} \stackrel{\mathrm{id}}{\longrightarrow} \mathfrak{t} & \text { on objects }, \\
(x, m, z) & \longmapsto(x, m, z \cdot \exp (J(x, m))) & \text { on arrows },
\end{array}
$$

together with the natural transformation

$$
\phi: F(-) \bullet_{J}^{t} F(-) \Longrightarrow F\left(-\bullet_{J}-\right)
$$

with

$$
\phi_{x, y}: x+y \stackrel{\exp (J(x, y))}{\longrightarrow} x+y \text {. }
$$

\section{Corollary 2.8.}

(i) If I is an even symmetric bilinear form on $\Lambda^{\vee}$, then the multiplicative bundle gerbe associated to I possesses a square root.

(ii) If $B$ is a skew symmetric integral bilinear form on $\Lambda^{\vee}$, then $B$ yields a trivial 2-group extension of $T$.

Proof. Claim (i) follows from Proposition 2.7, using Remark 2.5 and the fact that every even symmetric bilinear form $I$ can be written in the form $I=J+J^{t}$ for an integer-valued bilinear form $J$. For instance, fix a basis $\left(b_{1}, \ldots, b_{r}\right)$ of $\Lambda^{\vee}$ and set

$$
J\left(b_{i}, b_{j}\right)= \begin{cases}\frac{1}{2} I\left(b_{i}, b_{i}\right) & \text { if } i=j \\ I\left(b_{i}, b_{j}\right) & \text { if } i<j \\ 0 & \text { else. }\end{cases}
$$

Claim (ii) follows, similarly, from the fact that every skew symmetric bilinear form $B$ can be written in the form $B=J-J^{t}$ for an integer-valued bilinear form $J$.

Corollary 2.9. Let $J$ be an integer-valued bilinear form on $\Lambda^{\vee}$. Then, up to equivalence, the categorical extension $\left(\mathcal{T}, \bullet_{J}\right)$ of $T$ by $\mathrm{pt} / / U(1)$ only depends on the even bilinear form

$$
I(m, n)=J(m, n)+J(n, m) .
$$

Proof. Let $J_{1}$ and $J_{2}$ be two integer-valued bilinear forms on $\Lambda^{\vee}$, and assume that

$$
J_{1}+J_{1}^{t}=J_{2}+J_{2}^{t} .
$$

Then $J_{1}-J_{2}$ is skew symmetric. By Corollary 2.8 , it follows that the multiplicative bundle gerbe obtained from $J_{1}-J_{2}$ is trivial. Using Remark 2.5, we conclude that the multiplicative bundle gerbes obtained from $J_{1}$ and $J_{2}$ are isomorphic.

\section{The example of the circle}

Let $\Lambda^{\vee}=\mathbb{Z}$ and $J(m, n)=m n$. The basic circle extension $\mathcal{U}(1)$ of the circle group $U(1)$ consists of the following data:

(i) the trivial bundle gerbe over $U(1)$, 
(ii) the line bundle $\mathbf{L}$ on $U(1) \times U(1)=\mathbb{R}^{2} / \mathbb{Z}^{2}$ defined by the multipliers

$$
\begin{aligned}
f_{(m, n)}: \mathbb{R}^{2} & \longrightarrow U(1), \\
(x, y) & \longmapsto \exp (m y)
\end{aligned}
$$

for $(m, n) \in \mathbb{Z}^{2}$,

(iii) the canonical isomorphism

$$
\alpha: \quad \mathrm{m}_{12}^{*} \mathbf{L} \otimes \operatorname{pr}_{12}^{*} \mathbf{L} \cong \mathrm{m}_{23}^{*} \mathbf{L} \otimes \operatorname{pr}_{23}^{*} \mathbf{L}
$$

over $U(1) \times U(1) \times U(1)$.

For $k \in \mathbb{Z}$, the $k^{\text {th }}$ circle extension $\mathcal{U}(1)_{k}$ of $U(1)$ is obtained by replacing the multipliers with $\exp (k m y)$.

Remark 3.1. Recall that gerbes on $U(1)$ are classified, up to stable isomorphism, by their Dixmier-Douady class in $H^{3}(U(1) ; \mathbb{Z})=0$. So, any bundle gerbe over $U(1)$ is trivializable. A multiplicative structure on the trivial bundle gerbe over $U(1)$ consists of a line bundle on $U(1) \times U(1)$ plus extra data, encoding associativity. Line bundles on $U(1) \times U(1)$ are classified, up to isomorphism, by their first Chern class in

$$
H^{2}(U(1) \times U(1) ; \mathbb{Z}) \cong \operatorname{Alt}^{2}\left(\mathbb{Z}^{2}\right),
$$

the group of skew symmetric bilinear forms on $\mathbb{Z}^{2}$. This group is infinite cyclic, generated by the determinant, and we claim $c_{1}(\mathbf{L})=-$ det. This fact is proved using Chern-Weil theory, as illustrated in Fig. 1:

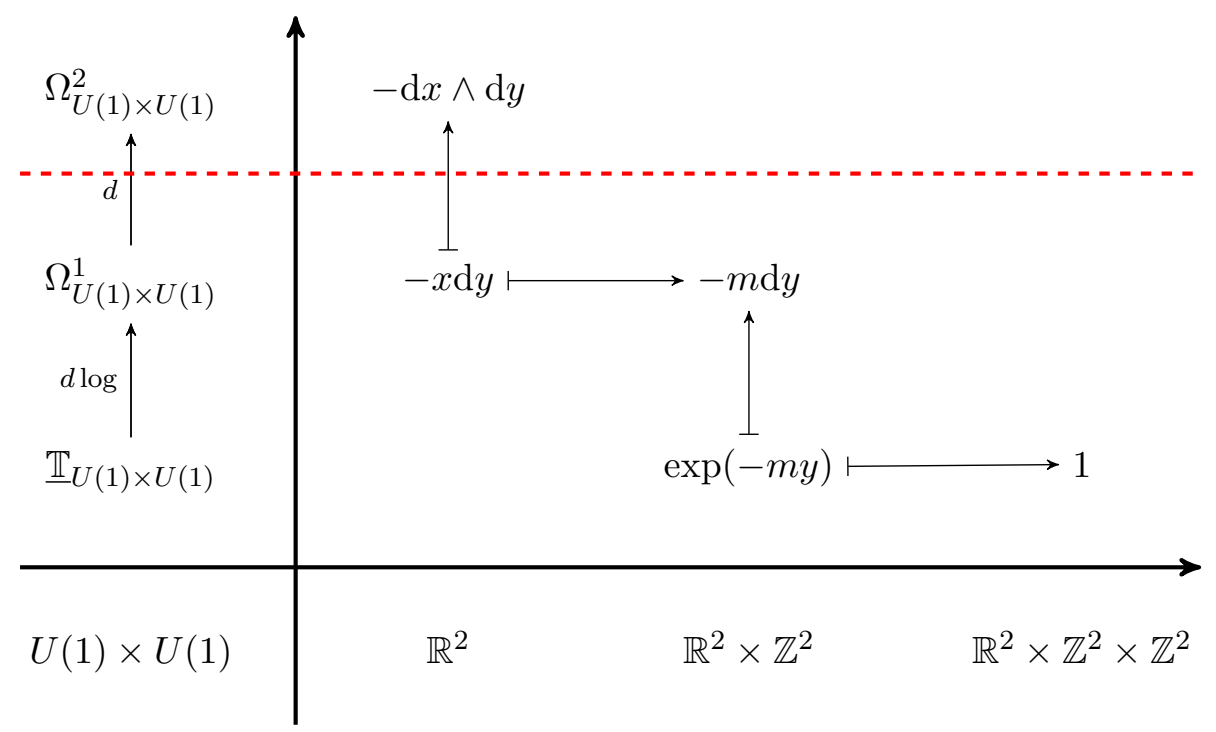

Figure 1. This 2-cocycle in the truncated Čech-de Rham double complex relates the multipliers of $\mathbf{L}$ to the 2 -form - det.

The entry $\exp (-m y)$ is the transition function of the bundle $\mathbf{L}$ for the cover $\mathbb{R}^{2} \rightarrow \mathbb{R}^{2} / \mathbb{Z}^{2}$. This convention, that the transition functions in Čech cohomology are taken to be the inverse multipliers, comes from algebraic geometry: with the identifications $\mathbb{R}^{2} \cong \mathbb{C}$ and $\mathbb{Z}^{2} \cong\langle 1, \tau\rangle$, the line bundle $\mathbf{L}$ is the topological line bundle underlying any degree -1 line bundle on the elliptic 
curve $\mathbb{C} /\langle 1, \tau\rangle$. Note also that this argument equips $\mathbf{L}$ with a connection, namely the one with 1 -form

$$
\omega=-x \mathrm{~d} y
$$

on $\mathbb{R} \times \mathbb{R}$ and with curvature

$$
\mathrm{d} \omega=-\mathrm{d} x \wedge \mathrm{d} y .
$$

Similarly, the bundle $\mathbf{L}^{\otimes k}$ with multipliers $\exp (k m y)$ has connection $k x d y$ and Chern class $k \cdot$ det.

Remark 3.2. We refer the reader to [3, Theorem 2.2.11] for a detailed discussion of line bundles with connection in this setting. This example is not new. A construction of the multiplicative gerbe $\mathcal{U}(1)$ (but with a different connection) already turns up in [4, Section 3]. A construction of $\mathcal{U}(1)$ as equivariant bundle gerbe is given in [9]

\section{The classification}

Recall from [12] that, up to equivalence, the 2-group extensions of $T$ by pt //U(1) are classified by degree three Lie group cohomology classes of $T$ with coefficients in $U(1)$. There are a number of constructions of Lie group cohomology, and a unifying axiomatic framework was recently given in [14]. We choose to work with the Cech-simplicial double complex of [3] and [5, Proposition 5.2]. So, the relevant cohomology group is

$$
H_{g p}^{3}(B T ; U(1))=\check{H}^{3}\left(B T_{\bullet} ; U(1)\right) .
$$

The goal of this section is to analyze the composite of isomorphisms

$$
S^{*}(\Lambda) \cong H^{2 *}(B T ; \mathbb{Z}) \cong \check{H}^{2 *}\left(B T_{\bullet} ; \mathbb{Z}\right) \cong \check{H}^{2 *-1}\left(B T_{\bullet} ; U(1)\right)
$$

in degree $*=2$. Here $S^{*}(\Lambda)$ is the symmetric algebra of the weight lattice $\Lambda=\operatorname{Hom}\left(\Lambda^{\vee}, \mathbb{Z}\right)$. We may think of elements of $S^{2}(\Lambda)=(\Lambda \otimes \Lambda) / S_{2}$ as homogeneous polynomials of degree 2 in the weights, and we have the symmetrization map

$$
\begin{aligned}
S^{2}(\Lambda) & \longrightarrow \operatorname{Bil}\left(\Lambda^{\vee}, \mathbb{Z}\right), \\
\lambda \mu & \longmapsto \lambda \otimes \mu+\mu \otimes \lambda,
\end{aligned}
$$

identifying $S^{2}(\Lambda)$ with the group of even symmetric bilinear forms on $\Lambda^{\vee}$.

Theorem 4.1. Let $I$ be an even symmetric bilinear form on $\Lambda^{\vee}$, and let $J$ be an integral bilinear form on $\Lambda^{\vee}$ satisfying $I=J+J^{t}$. Then I classifies the multiplicative bundle gerbe obtained from $\left(\Lambda^{\vee},-J\right)$ via Construction 2.3.

Proof. Let $E T$ be a contractible space on which $T$ acts freely, and let $B T=E T / T$ be our model for the clasifying space of $T$. On $B T$, we have the line bundles

$$
L_{\lambda}=E T \times_{T} \mathbb{C}_{\lambda} \cong\left(E T \times \mathbb{C}_{-\lambda}\right) / T,
$$

$\lambda \in \Lambda$, where $\mathbb{C}_{\lambda}$ denotes the irreducible complex representation of $T$ with weight $\lambda$. The first isomorphism in (4.1) is

$$
\begin{aligned}
S^{*}(\Lambda) & \stackrel{\cong}{\longrightarrow} H^{2 *}(B T ; \mathbb{Z}), \\
\lambda & \longmapsto c_{1}\left(L_{\lambda}\right) .
\end{aligned}
$$


Its definition goes back to Borel, and it is an isomorphism of graded rings. It is, of course, well known that the odd cohomology groups vanish, but this fact will not concern us here. To define the second isomorphism in (4.1), we use the homeomorphism

$$
\begin{aligned}
E T \times T \times \cdots \times T & \cong E T \times_{B T} \cdots \times_{B T} E T, \\
\left(e, t_{1}, \ldots, t_{n}\right) & \longmapsto\left(e, t_{1} e, \ldots, t_{n} e\right)
\end{aligned}
$$

to interpret the maps

$$
\begin{aligned}
E T & \longrightarrow B T, \\
E T \times \mathfrak{t} & \longrightarrow E T \times_{B T} E T
\end{aligned}
$$

as a hypercover of $B T$. The Čech double complex of this hypercover can be identified with the Čech simplicial double complex $\check{C}^{*}\left(B T_{\bullet} ; \mathbb{Z}\right)$. Under this identification, the cup product becomes

$$
(f \cup g)\left(x_{0}, \ldots, x_{r+s}\right)=\left(\operatorname{pr}_{1}^{*} f\right)\left(x_{0}, \ldots x_{r}\right) \cdot\left(\operatorname{pr}_{2}^{*} g\right)\left(x_{r}, \ldots x_{r+s}\right),
$$

where $r$ and $s$ are the Čech degrees of $f$ and $g$, while $\operatorname{pr}_{1}$ is the projection onto the first $\operatorname{deg}_{\text {simp }}(f)$ factors, and $\mathrm{pr}_{2}$ is the projection onto the last $\operatorname{deg}_{\text {simp }}(g)$ factors. To determine the image of $\lambda \mu$ in $H^{4}\left(B T_{\bullet} ; \mathbb{Z}\right)$, we determine the images of $\lambda$ and $\mu$ and then take the cup product. The first Chern class of $L_{\lambda}$ in Čech hypercohomology is obtained by applying $\delta_{\check{C} \text { ech }} \log$ to the inverse multipliers

$$
\begin{aligned}
E T \times \mathfrak{t} & \longrightarrow U(1), \\
(\eta, x) & \longmapsto \exp (\lambda(x))
\end{aligned}
$$

of $L_{\lambda}$. So, $c_{1}\left(L_{\lambda}\right)$ is represented by the degree $(1,1)$ cocycle

$$
\begin{aligned}
\mathfrak{t} \times \Lambda^{\vee} & \longrightarrow \mathbb{Z}, \\
(x, m) & \longmapsto \lambda(m)
\end{aligned}
$$

in the Čech-simplicial double complex. Given two weights, $\lambda$ and $\mu$, their cup product is represented by the cocycle

$$
\begin{gathered}
\mathfrak{t}^{2} \times\left(\Lambda^{\vee}\right)^{2} \times\left(\Lambda^{\vee}\right)^{2} \longrightarrow \mathbb{Z}, \\
\left(\left(\begin{array}{l}
x \\
y
\end{array}\right),\left(\begin{array}{c}
m \\
n
\end{array}\right),\left(\begin{array}{l}
k \\
l
\end{array}\right)\right) \longmapsto \lambda(k) \cdot \mu(n)
\end{gathered}
$$

in $\check{C}^{2}\left(B T_{2} ; \mathbb{Z}\right)$. The last isomorphism in (4.1) is the inverse of the connecting homomorphism for the short exact sequence of presheaves

$$
0 \longrightarrow \mathbb{Z} \longrightarrow \underline{\mathbb{R}} \longrightarrow \underline{U(1)} \longrightarrow 0 .
$$

Let $J=\mu \otimes \lambda$. As in Remark 3.1, the transition function for $\mathbf{L}_{-J}$ is the inverse multiplier. So, Construction 2.3 associates to $-J$ the multiplicative bundle gerbe corresponding to the $U(1)$-valued Čech-simplicial 3-cocycle $(1, \lambda(m) \mu(y), 1,1)$. The image of this cocycle under the connecting homomorphism is the integral 4-cocycle

$$
(1,1, \lambda(k) \mu(n), 1,1)=c_{1}\left(L_{\lambda}\right) \cdot c_{1}\left(L_{\mu}\right) .
$$

The general case now follows from Remark 2.5 together with the fact that any choice of $J$ can be written as linear combination of summands of the form $\mu \otimes \lambda$. 


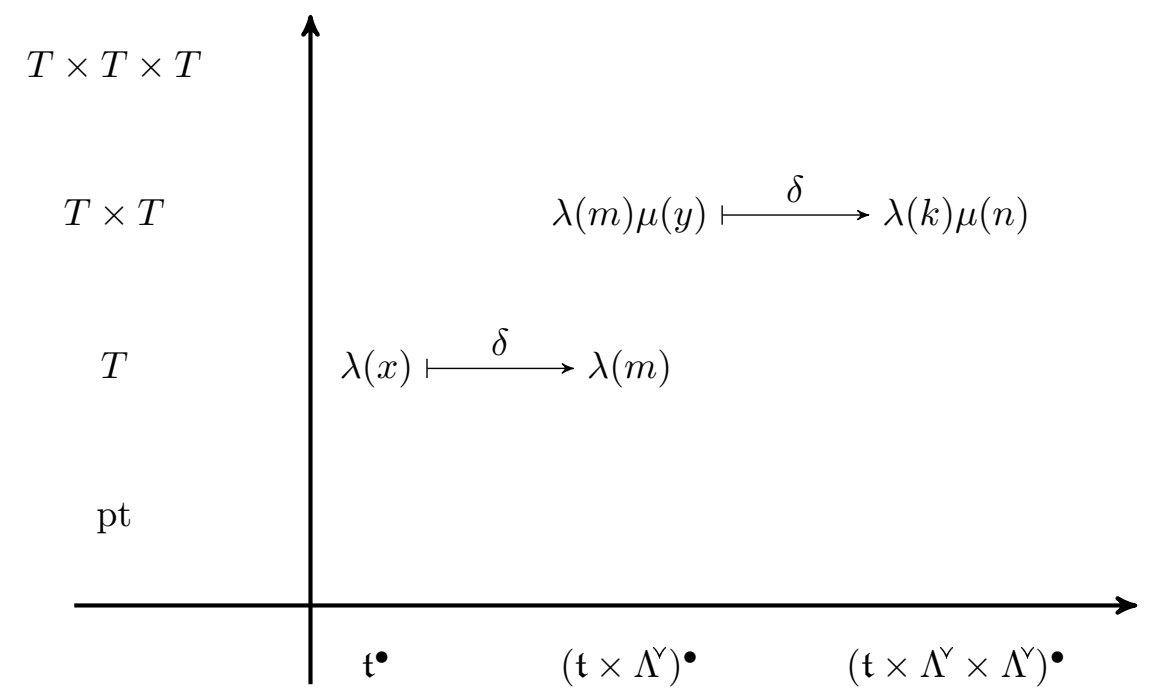

Figure 2. $\delta_{\breve{\text { C̆ech }}}: \check{C}^{1}\left(B T_{2} ; \underline{\mathbb{R}}\right) \longrightarrow \check{C}^{2}\left(T_{2} ; \underline{\mathbb{R}}\right)$.

Remark 4.2. In particular, we have seen that the underlying gerbe of any multiplicative bundle gerbe on $T$ is trivial. This is consistent with work of Waldorf [15, Proposition 2.10], who identifies the forgetful map

$\{$ multiplicative bundle gerbes on $G\} / \cong \longrightarrow\{$ bundle gerbes on $G\} / \cong$

with the inverse transgression $\tau: H^{4}(B G ; \mathbb{Z}) \longrightarrow H^{3}(G ; \mathbb{Z})$ in the Leray-Serre spectral sequence for the fibration $E G \rightarrow B G$. For a compact, connected Lie group $G$, this inverse transgression map was calculated (in all degrees) by Chern and Simons. In the relevant degrees, their result is summarized by the commuting diagram ${ }^{1}$

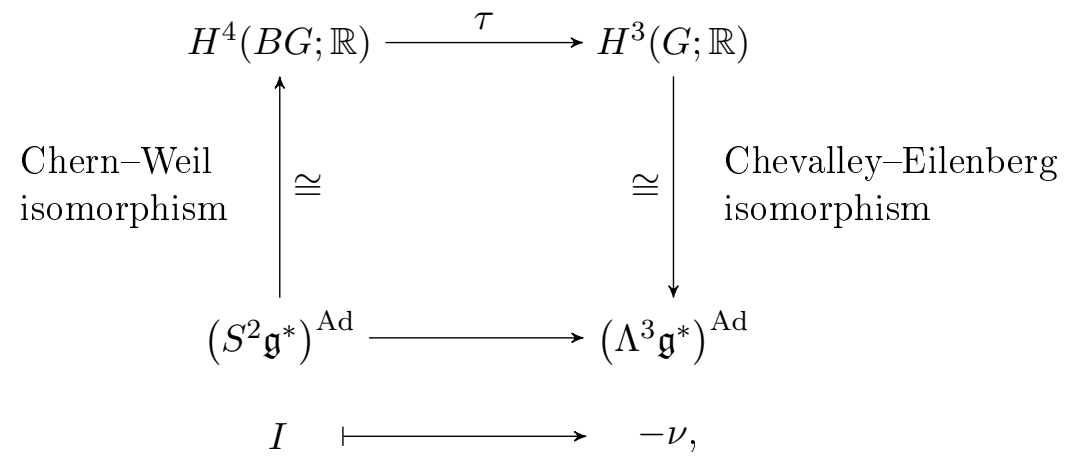

where $\nu$ is the Cartan 3 -form associated to $I$,

$$
\nu(x, y, z)=I([x, y], z) .
$$

So, the multiplicative bundle gerbe on $G$ classified by $I$ has as its Dixmier-Douady class the left-invariant 3-form on $G$ whose restriction to $\mathfrak{g}$ is $-\nu$. Since the Lie bracket on a torus is zero, it follows that the Dixmier-Douady class of any multiplicative bundle gerbe on $T$ vanishes.

${ }^{1}$ The formula [6, equation (3.10)] is often cited in its original form

$$
I \longmapsto-\frac{1}{12} I(\omega \wedge[\omega, \omega]),
$$

where $\omega$ is the right-invariant Maurer-Cartan form on $G$. A look at the definitions on p. 50 of [6] identifies $I(\omega \wedge[\omega, \omega])$ with the bi-invariant 3 -form on $G$ whose restriction to $\mathfrak{g}=T_{1} G$ equals $12 \nu$. 


\subsection{Connections}

Let $\mathbf{L}$ be the line bundle of Construction 2.3. If $J=\lambda \otimes \mu$, then we have the connection $\nabla$ on $\mathbf{L}$ whose 1 -form on $\mathfrak{t} \times \mathfrak{t}$ is

$$
\omega=-\lambda \mathrm{d} \mu \text {. }
$$

It has curvature 2 -form

$$
\kappa=-\mathrm{d} \lambda \wedge \mathrm{d} \mu \text {. }
$$

For arbitrary $J$, we introduce the maps

$$
\begin{aligned}
J^{\sharp}(x): \mathfrak{t} & \rightarrow \mathbb{R}, \\
v & \longmapsto J(x, v) .
\end{aligned}
$$

Then $\nabla$ is defined by the 1 -form

$$
\omega_{(x, y)}=-\mathrm{d}\left(J^{\sharp}(x)\right)_{y} .
$$

The pair $(\mathbf{L}, \nabla)$ turns the trivial bundle gerbe $\mathcal{I}$ on $T$ into a multiplicative bundle gerbe with connection (in the sense of [15], with the remaining data trivial).

\section{$5 \quad$ Examples}

\subsection{Maximal tori}

Let $G$ be a simple and simply connected compact connected Lie group with maximal torus $T$ and Weyl group $W$. Then we have

$$
\mathbb{Z} \cong H_{\mathrm{gp}}^{3}(G ; U(1)) \cong H^{4}(B T ; \mathbb{Z})^{W} \cong\left(\operatorname{Bil}_{\mathrm{ev}}\left(\Lambda^{\vee} ; \mathbb{Z}\right)^{S_{2}}\right)^{W}
$$

The elements of this group are multiples of the Killing form, and the positive definite generator $I_{\text {basic }}$ classifies the Lie 2-group extension of $G$ denoted $\operatorname{String}(G)$ in [12]. We arrive at a recognition principle for these 2-groups: the extension $\mathcal{G}$ is equivalent to $\operatorname{String}(G)$ if and only if its restriction to $T$ is equivalent to the categorical torus classified by $\left(\Lambda^{\vee}, I_{\text {basic }}\right)$.

\subsection{The Leech lattice}

Another interesting example is given by the Leech lattice $\Lambda^{\vee}=\Lambda_{\text {Leech }}$ inside $\mathbb{R}^{24}$, together with the standard symmetric bilinear form $I$. The group of linear isometries $O\left(\Lambda^{\vee}, I\right)$ of the Leech lattice is the Conway group $\mathrm{Co}_{0}$. In analogy to the previous example, we view $I$ as a $\mathrm{Co}_{0}$-invariant cohomology element,

$$
I \in H^{4}(B T, \mathbb{Z})^{\mathrm{Co}_{0}},
$$

where $T=\mathbb{R}^{24} / \Lambda_{\text {Leech }}$ is the Leech torus. We arrive at a categorical extension $\mathcal{T}_{\text {Leech }}$ of the Leech torus, on which $\mathrm{Co}_{0}$ acts by autoequivalences. It is now understood [8] that the Conway group has a universal categorical central extension by the cyclic group with 24 elements, which we believe to be closely related, but not equal to the symmetries of the categorical Leech torus. 


\subsection{Niemeyer lattices}

Similarly, we can choose $\Lambda^{\vee}$ as one of the Niemeyer lattices, and $I$ as the standard symmetric bilinear form on $\mathbb{R}^{24}$, making $\Lambda^{\vee}$ an even unimodular lattice. If $\Lambda^{\vee}$ equals $A_{1}^{24}$ or $A_{2}^{12}$, then the group of linear isometries of $\left(\Lambda^{\vee}, I\right)$ is the Mathieu group $M_{24}$ (respectively $M_{12}$ ), and we have

$$
I \in H^{4}(B T, \mathbb{Z})^{M_{24}} \quad \text { respectively } \quad I \in H^{4}(B T, \mathbb{Z})^{M_{12}},
$$

classifying two categorical tori on which the respective Mathieu groups act by autoequivalences.

\section{Extraspecial categorical 2-groups}

Assume we are given a strict action of a group $G$ by functors on a category $\mathcal{C}$. In other words, assume that $\mathcal{C}$ comes equipped with endofunctors $\varrho(g)$, one for each $g \in G$, satisfying

$$
\varrho(g) \varrho(h)=\varrho(g h) \quad \text { and } \quad \varrho(1)=\mathrm{id} .
$$

In this situation, we will allow ourselves to drop $\varrho$ from the notation and simply write $g$ for the functor $\varrho(g)$.

Definition 6.1 (Grothendieck). An equivariant object of $\mathcal{C}$ is a pair

$$
(x, \mathbf{e})=\left(x,\left\{e_{g}\right\}_{g \in G}\right),
$$

where $x \in \mathrm{ob}(\mathcal{C})$ and the $e_{g}: x \stackrel{\cong}{\longrightarrow} g x$ are isomorphisms in $\mathcal{C}$ satisfying

$$
\left(g e_{h}\right) \circ e_{g}=e_{g h}
$$

for all $g$ and $h$ in $G$. An equivariant arrow from $(x, \mathbf{e})$ to $(y, \mathbf{f})$ is an arrow $a: x \rightarrow y$ in $\mathcal{C}$ that is compatible with $\mathbf{e}$ and $\mathbf{f}$ in the following sense

$$
f_{g} \circ a=(g a) \circ e_{g}
$$

for all $g \in G$.

Definition 6.2. We will refer to the category $\mathcal{C}^{G}$ of equivariant objects of $\mathcal{C}$ and equivariant arrows between them as the categorical fixed points $\mathcal{C}^{G}$ of the action of $G$ on $\mathcal{C}$.

The goal of this section is to identify the fixed point category of the action of $\{ \pm 1\}$ on $\mathcal{T}$, where -1 acts by the auto-equivalence associated to the crossed module automorphism

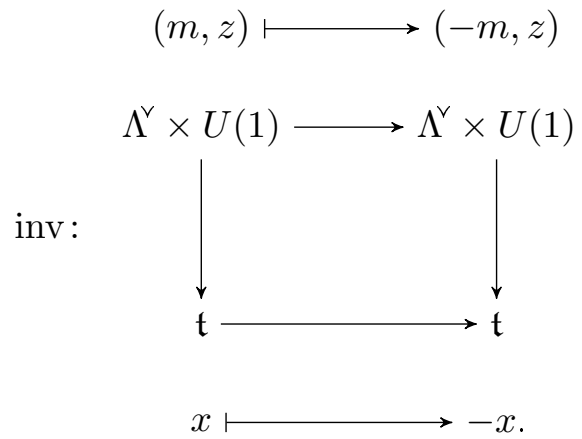

Explicitly, the functor $\varrho(-1)$ sends the object $x$ to $-x$ and the arrow $x \stackrel{z}{\longrightarrow} x+m$ to the arrow $-x \stackrel{z}{\longrightarrow}-x-m$. Since this is an action by strictly monoidal functors, the categorical fixed 
points $\mathcal{T}^{\{ \pm 1\}}$ inherit the structure of a strict categorical group. Consider now the points of order two in $T$. These form the elementary abelian 2 -group ${ }^{2}$

$$
T[2] \cong \Lambda^{\vee} \otimes \mathbb{F}_{2} .
$$

We have a central extension $\widetilde{T[2]}$ of $T[2]$, defined by the $\mathbb{F}_{2}$-valued 2-cocycle

$$
J_{\mathbb{F}_{2}}:=J \otimes \mathbb{F}_{2} .
$$

Central extensions of this form are know as extraspecial 2-groups and classified by their Arf invariant, i.e., by the quadratic form

$$
v \longmapsto J_{\mathbb{F}_{2}}(v, v)
$$

on the $\mathbb{F}_{2}$-vector space $\Lambda^{\vee} \otimes \mathbb{F}_{2}$. Writing its center multiplicatively, our central extension takes the form

$$
1 \longrightarrow\{ \pm 1\} \longrightarrow \widetilde{T[2]} \longrightarrow T[2] \longrightarrow 1
$$

Similarly, we have the (non-canonically trivial) central extension

$$
1 \longrightarrow\{ \pm 1\} \longrightarrow \widetilde{\Lambda}^{\vee} \longrightarrow \Lambda^{\vee} \longrightarrow 1
$$

with 2-cocycle

$$
(m, n) \longmapsto(-1)^{J(m, n)} .
$$

Theorem 6.3. The crossed module corresponding to the strict categorical group $\mathcal{T}^{\{ \pm 1\}}$ is isomorphic to the crossed module

$$
\begin{aligned}
\Sigma: \Lambda^{\vee} \times U(1) & \longrightarrow \widetilde{\Lambda}, \\
(m, z) & \longmapsto(2 m, 1),
\end{aligned}
$$

where $(n, \varepsilon) \in \widetilde{\Lambda^{\vee}}$ acts on $\Lambda^{\vee} \times U(1)$ by

$$
(m, z) \longmapsto\left(m, z \cdot(-1)^{J(m, n)}\right) .
$$

In particular, $\mathcal{T}^{\{ \pm 1\}}$ is part of a categorical central extension

$$
1 \longrightarrow \mathrm{pt} / / U(1) \longrightarrow \mathcal{T}^{\{ \pm 1\}} \longrightarrow \widetilde{T[2]} \longrightarrow 1
$$

Proof. For $\mathcal{T}^{\{ \pm 1\}}$, condition (6.1) reads

$$
e_{1}=\operatorname{id}_{x} \quad \text { and } \quad \operatorname{inv}\left(e_{-1}\right) \circ e_{-1}=\mathrm{id}_{x} .
$$

So, we have a fully faithful and strictly monoidal embedding

$$
\begin{aligned}
\mathcal{T}^{\{ \pm 1\}} \longrightarrow \mathcal{T}^{I} \\
(x, e) \longmapsto e_{-1},
\end{aligned}
$$

\footnotetext{
${ }^{2}$ In the context of this section, the term 2-group is used in the sense of $p$-group, $p$ a prime, not in the sense of categorical group.
} 
where $\mathcal{T}^{I}$ denotes the arrow category of $\mathcal{T}$. This embedding identifies the objects of $\mathcal{T}^{\{ \pm 1\}}$ with the image of the injective group homomorphism

$$
\begin{aligned}
\widetilde{\Lambda^{\vee}} & \longrightarrow \operatorname{ob}\left(\mathcal{T}^{I}\right), \\
(m, \varepsilon) & \longmapsto\left(-\frac{m}{2} \stackrel{\varepsilon}{\longrightarrow} \frac{m}{2}\right) .
\end{aligned}
$$

The kernel of the source of $\mathcal{T}^{\{ \pm 1\}}$ is identified with the image of the group homomorphism

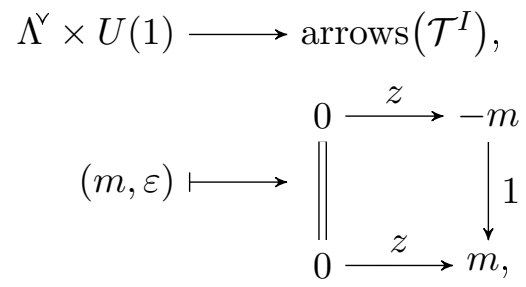

and under these identifications, the target map becomes the homomorphism $\Sigma$ of the theorem. It remains to identify the conjugation action of the objects on the kernel of the source. We have

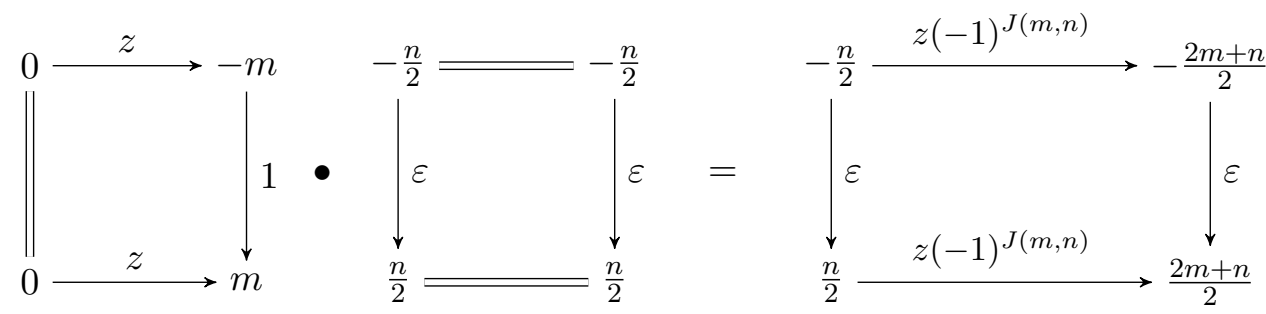

and
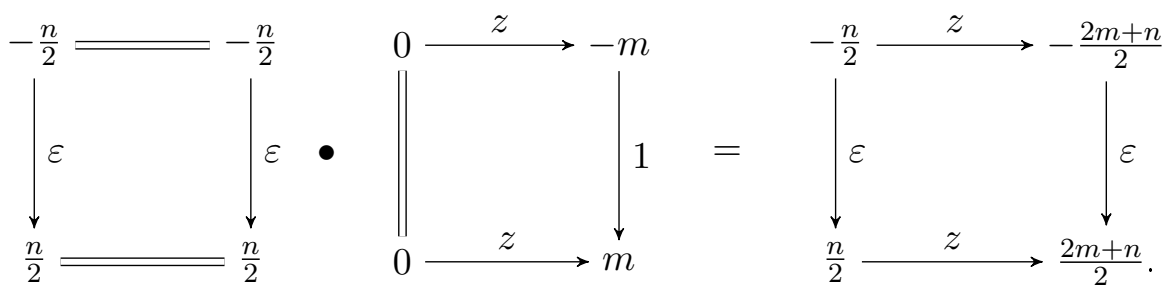

So, the conjugation action is as claimed.

Remark 6.4. Let $I=J+J^{t}$, and consider the integer-valued quadratic form

$$
\phi(m)=\frac{1}{2} I(m, m)
$$

on $\Lambda^{\vee}$. Its reduction mod 2 is the Arf invariant of $\widetilde{T[2]}$. The form $I$ can be recovered from $\phi$ by the identity

$$
I=\delta_{\text {simp }}(\phi),
$$

i.e.,

$$
I(m, n)=\phi(m+n)-\phi(m)-\phi(n) .
$$

Example 6.5. There is a prominent subgroup $C$ of the Monster, sitting in a non-split extension

$$
1 \longrightarrow \widetilde{T[2]} \longrightarrow \mathrm{C} \longrightarrow \mathrm{Co}_{1} \longrightarrow 1
$$

where $\mathrm{Co}_{1}$ is the Conway group $\mathrm{Co}_{1}=\mathrm{Co}_{0} / \pm 1$. This subgroup is typically the first step in the construction of the Monster, see for instance [13] or [7]. 


\section{From categorical groups to loop groups}

The relationship between loop groups and categorical Lie groups has been the subject of extensive study. In [2], the authors use central extensions of loop groups to define 2-groups. We will mostly be interested in the other direction and work with the transgression-regression machine of $[16,17]$. In this context, the term 'transgression' refers to a recipe for turning multiplicative bundle gerbes with connection into central extensions of loop groups. Let $\mathcal{L} T$ be the group of piecewise smooth loops in $T$. This itself is not a manifold, but it is densely isomorphic to an increasing union of manifolds of loops which are smooth over a given subdivision of $\mathbb{S}^{1}$ into intervals. Here (and similarly below), we will follow Brylinski [3, p. 96] and call a map on $\mathcal{L} T$ smooth if its restriction to each such manifold is smooth. Applied to the trivial bundle gerbe $\mathcal{I}$ on $T$ equipped with the trivial connection, transgression yields the trivial principal $U(1)$-bundle over $\mathcal{L} T$. If we further apply transgression to our multiplicative structure with connection $((\mathbf{L}, \nabla), \alpha)$, we obtain the central extension of $\mathcal{L} T$ whose 2-cocycle $c$ is given by the holonomy of $(\mathbf{L}, \nabla)$. So, if $\phi$ and $\gamma$ are loops in $T$, and $(f, g)$ is any choice of lift of $(\varphi, \gamma)$ to $\mathfrak{t} \times \mathfrak{t}$, then we have

$$
c(\varphi, \gamma)=\operatorname{Hol}_{(\mathbf{L}, \nabla)}((\varphi, \gamma))=\exp \left(\left(\int_{0}^{1} J(f(t), \dot{g}(t)) \mathrm{d} t\right)-J\left(\Delta_{f}, g(0)\right)\right),
$$

where $\Delta_{f}=f(1)-f(0)$. This formula results from a variation of [3, Proposition 6.1.3]. The underlying principal bundle of this central extension is trivial.

Lemma 7.1. The central extension $\widetilde{\mathcal{L} T}$ defined by $c$ is isomorphic to that in [11, Section 4.8].

Proof. Pressley and Segal denote our $I$ by $\langle-,-\rangle$, their $b$ can be taken to be our $J$, and they write $\Lambda$ for the cocharacter lattice

$$
\check{T}=\operatorname{Hom}(U(1), T) \text {. }
$$

Our proof follows closely that of [11, Proposition 4.8.3]. We first note that $c$ describes the correct extension of this lattice

$$
\Lambda^{\vee} \cong \check{T} \subset \mathcal{L} T
$$

namely

$$
c_{\Lambda} \vee(m, n)=(-1)^{J(m, n)} .
$$

The commutator $\widetilde{\varphi} \cdot \widetilde{\gamma} \cdot \widetilde{\varphi}^{-1} \cdot \widetilde{\gamma}^{-1}$ in $\widetilde{\mathcal{L} T}$ equals

$$
\begin{aligned}
\frac{c(\varphi, \gamma)}{c(\gamma, \varphi)} & =\exp \left(\int_{0}^{1} J(f(t), \dot{g}(t)) \mathrm{d} t-\int_{0}^{1} J(g(t), \dot{f}(t)) \mathrm{d} t-J\left(\Delta_{f}, g(0)\right)+J\left(\Delta_{g}, f(0)\right)\right) \\
& =\exp \left(\int_{0}^{1} I(f(t), \dot{g}(t)) \mathrm{d} t-[J(g(t), f(t))]_{0}^{1}-J\left(\Delta_{f}, g(0)\right)+J\left(\Delta_{g}, f(0)\right)\right) \\
& =\exp \left(\int_{0}^{1} I(f(t), \dot{g}(t)) \mathrm{d} t-J\left(g(1), \Delta_{f}\right)-J\left(\Delta_{f}, g(0)\right)\right) \\
& =\exp \left(-\int_{0}^{1} I(\dot{f}(t), g(t)) \mathrm{d} t+[J(f(t), g(t))]_{0}^{1}-J\left(\Delta_{f}, g(0)\right)+J\left(\Delta_{g}, f(0)\right)\right) \\
& =\exp \left(-\int_{0}^{1} I(\dot{f}(t), g(t)) \mathrm{d} t+J\left(f(1), \Delta_{g}\right)+J\left(\Delta_{g}, f(0)\right)\right) .
\end{aligned}
$$


The second and fourth equality are obtained from the first line through integration by parts. Over the identity component of $\mathcal{L} T$, our extension is completely described by its Lie-algebra cocycle,

$$
\omega=\int_{0}^{1} I(f(t), \dot{g}(t)) \mathrm{d} t
$$

(from line 3 and [11, Section 2.1]), which agrees with the expression for $\omega$ in [11, Proposition 4.2.2]. The adjoint action of $\varphi \in \mathcal{L} T$ on $\widetilde{L t}$ can be read off from the last line. It sends $(g, r) \in \widetilde{L t}$ to

$$
\left(g, r-\int_{0}^{1} I(\dot{f}(t), g(t)) \mathrm{d} t\right) .
$$

This agrees with the expression in [11, Proposition 4.3.2].

Remark 7.2. This definition of $\widetilde{\mathcal{L T}}$, as transgression of a categorical torus, leads to a considerable simplification of the picture in the standard literature on loop groups [11, Section 4.8]. The expression (7.1) is less complicated than [11, Proposition 4.8.3]. It is, by construction, invariant under the action of $\operatorname{Diff}^{+}\left(\mathbb{S}^{1}\right)$. There is no need to restrict ourselves to maximal tori of simply laced groups. Further, the cocycle $c$ satisfies the fusion rule

$$
c\left(\varphi_{1} * \bar{\varphi}_{2}, \gamma_{1} * \bar{\gamma}_{2}\right) \cdot c\left(\varphi_{2} * \bar{\varphi}_{3}, \gamma_{2} * \bar{\gamma}_{3}\right)=c\left(\varphi_{1} * \bar{\varphi}_{3}, \gamma_{1} * \bar{\gamma}_{3}\right) .
$$

Here $*$ stands for concatenation of paths, and $\left(\varphi_{1}, \varphi_{2}, \varphi_{3}\right)$ and $\left(\gamma_{1}, \gamma_{2}, \gamma_{3}\right)$ are triples of paths with identical start and end points, i.e.,

$$
\varphi_{1}(0)=\varphi_{2}(0)=\varphi_{3}(0) \quad \text { and } \quad \varphi_{1}(1)=\varphi_{2}(1)=\varphi_{3}(1)
$$

and likewise for the $\gamma_{i}$.

Our fourth construction of $\mathcal{T}$ applies Waldorf's regression machine to reconstruct our categorical torus from the cocycle $c$.

Construction 7.3. Given $\left(\Lambda^{\vee}, J\right)$, we let $T$ be the torus $\Lambda^{\vee} \otimes U(1)$ and $\widetilde{\mathcal{L} T}$ the central extension of the loop group $\mathcal{L} T$ defined in (7.1). We write $\mathcal{P}_{1} T$ for the space of piecewise smooth paths based at 1 in $T$ and

$$
\Omega T \cong \mathcal{P}_{1} T \times_{T} \mathcal{P}_{1} T
$$

for the group of piecewise smooth loops based at 1 . Then we have the groupoid

$$
\mathcal{G}:=\left(\mathcal{P}_{1} T \longleftarrow \widetilde{\Omega T}\right),
$$

where the composition of arrows is

$$
\left(z, \gamma_{2}, \gamma_{3}\right) \circ\left(w, \gamma_{1}, \gamma_{2}\right)=\left(z w, \gamma_{1}, \gamma_{3}\right)
$$

The fusion rule (7.2) ensures that this composition is a group homomorphism, making $\mathcal{G}$ a groupoid in groups.

It is probably possible to articulate the manner in which this is an infinite-dimensional ind Lie-groupoid, but we will not bother, nor will we dive into the technicalities of diffeological spaces as in Waldorf. We are grateful to David Roberts for explaining the following way to work around smoothness issues: Fix an order preserving smooth map

$$
f:[0,1] \longrightarrow[0,2]
$$


sending $\frac{1}{2}$ to 1 . We ask that all derivatives of $f$ vanish at 0 , at $\frac{1}{2}$, and at 1 and that, away from these points, $f$ is a diffeomorphism onto $(0,1) \cup(1,2)$. Consider a pair of smooth paths $\varphi, \gamma:[0,1] \longrightarrow T$ with $\varphi(1)=\gamma(0)$. The (mollified) $f$-concatentation of $\varphi$ and $\gamma$ is defined as

$$
\varphi *_{f} \gamma= \begin{cases}\varphi(f(t)) & t \leq \frac{1}{2} \\ \gamma(f(t)-1) & t \geq \frac{1}{2}\end{cases}
$$

Once can now proceed as before, with $*$ replaced by $f$-concatenation, which is smoothly homotopic to ordinary concatenation but also a smooth map on the smooth function spaces. The reparametrization invariance of the cocycle $c$ ensures that the calculations remain the same. The space of composable pairs of smooth paths in Construction 7.3 is now identified with loops that are smooth away from possibly the basepoint and $\frac{1}{2}$. This is densly isomorphic to the space of smooth loops $\Omega^{\mathrm{sm}} T$. Moreover, the latter is a smooth retract of the former, as Lie groups. Waldorf's machine guarantees that Construction 7.3 recovers our categorical torus. We give an explicit equivalence in three steps.

Lemma 7.4. The strict categorical Lie group $\mathcal{G}$ of Construction 7.3 corresponds to the crossed module

$$
\begin{gathered}
\Pi: \widetilde{\Omega T} \longrightarrow \mathcal{P}_{1} T \\
(z, \gamma) \longmapsto \gamma,
\end{gathered}
$$

where $\widetilde{\Omega T}$ is the central extension with cocycle $c^{-1}$, equipped with the $\mathcal{P}_{1} T$-action

$$
(z, \gamma)^{\varphi}=(e z, \gamma)
$$

with

$$
e=\exp \left(\int_{0}^{1} I(f(t), \dot{g}(t)) \mathrm{d} t\right) .
$$

Here $(f, g)$ is the lift of $(\varphi, \gamma)$ to $\mathfrak{t} \times \mathfrak{t}$ that starts at $(0,0)$.

Proof. The kernel of the source of $\mathcal{G}$ consists of triples $(z, 1, \gamma)$ where $\gamma$ is a closed loop and 1 denotes the constant loop. Since $c$ is invariant under reparametrization, we have

$$
c(1 * \bar{\gamma}, 1 * \bar{\beta})=c(\bar{\gamma}, \bar{\beta})=c(\gamma, \beta)^{-1} .
$$

Similarly, the conjugation action of the loop $\varphi * \bar{\varphi}$ on $(z, 1, \gamma)$ is calculated as in the proof of Lemma 7.1.

In the following, we deviate from the notation in Section 6 and write $\widetilde{\Lambda}^{r}$ for the central extension of $\Lambda^{\vee}$ by $U(1)$ with cocycle $(-1)^{J}$. Recall that a (weak) map of crossed modules, in the sense of [10, Definition 8.4], is an equivalence if it induces a monoidal equivalence of the corresponding categorical groups.

Lemma 7.5. The crossed module of the previous lemma is equivalent to the crossed module

$$
\begin{aligned}
& \Xi: \widetilde{\Lambda}^{\vee} \longrightarrow \mathfrak{t}, \\
& (z, m) \longmapsto m
\end{aligned}
$$

with action

$$
(z, m)^{x}=\left(z \cdot \exp \left(\frac{1}{2} I(x, m)\right), m\right) .
$$


Proof. For $x \in \mathfrak{t}$, define the path

$$
\begin{aligned}
\gamma_{x}:[0,1] & \longrightarrow T, \\
t & \longmapsto \exp (t x)
\end{aligned}
$$

from 1 to $\exp (x)$ in $T$. The resulting group homomorphism from $\mathfrak{t}$ to $\mathcal{P}_{1} T$ identifies $\Lambda^{\vee}$ with the subgroup $\check{T} \subseteq \Omega T$, and makes $\Xi$ into an equivalent sub-crossed module of $\Pi$.

Lemma 7.6. We have an equivalence

$$
\Psi \simeq \Xi
$$

between the crossed module of Construction 2.1 and that of the previous lemma.

Proof. Let $a: \Lambda^{\vee} \longrightarrow\{ \pm 1\}$ be any 1 -cochain on $\Lambda^{\vee}$ with boundary $(-1)^{J}$. Then we have the group homomorphism

$$
\begin{aligned}
p_{2}: \Lambda^{\vee} \times U(1) & \longrightarrow \widetilde{\Lambda^{\vee}} \\
(m, z) & \longmapsto(z \cdot a(m), m) .
\end{aligned}
$$

Together with the maps $p_{1}=\mathrm{id}_{\mathfrak{t}}$ and

$$
\begin{aligned}
\varepsilon: \mathfrak{t} \times \mathfrak{t} & \longrightarrow \widetilde{\Lambda^{\vee}} \\
(x, y) & \longmapsto\left(\exp \left(\frac{1}{2} J(x, y)\right), 0\right),
\end{aligned}
$$

this defines a weak map of crossed modules inducing an equivalence of the corresponding categorical groups.

This concludes our argument that the four constructions of our categorical torus are equivalent.

\section{Acknowledgments}

The author was supported by an Australian Research Fellowship and by ARC grant DP1095815. It is a pleasure to thank David Roberts for very helpful conversations and correspondence as well as his open referee report. The idea for Construction 2.1 came from a conversation with him, and I understand that he will also write about it elsewhere. I would like to thank Shan Shah for pointing out a mistake in an earlier version. Many thanks for helpful and inspiring conversations also go to Matthew Ando, Konrad Waldorf, Thomas Nikolaus, Geoffrey Mason, Arun Ram, and Alex Ghitza. Finally I wish to thank the anonymous referee for helpful comments.

\section{References}

[1] Baez J.C., Huerta J., An invitation to higher gauge theory, Gen. Relativity Gravitation 43 (2011), 2335-2392, arXiv:1003.4485.

[2] Baez J.C., Stevenson D., Crans A.S., Schreiber U., From loop groups to 2-groups, Homology Homotopy Appl. 9 (2007), 101-135, math.QA/0504123.

[3] Brylinski J.-L., Loop spaces, characteristic classes and geometric quantization, Modern Birkhäuser Classics, Birkhäuser Boston, Inc., Boston, MA, 2008. 
[4] Brylinski J.-L., McLaughlin D.A., The geometry of degree-4 characteristic classes and of line bundles on loop spaces. II, Duke Math. J. 83 (1996), 105-139.

[5] Carey A.L., Johnson S., Murray M.K., Stevenson D., Wang B.-L., Bundle gerbes for Chern-Simons and Wess-Zumino-Witten theories, Comm. Math. Phys. 259 (2005), 577-613, math.DG/0410013.

[6] Chern S.S., Simons J., Characteristic forms and geometric invariants, Ann. of Math. 99 (1974), 48-69.

[7] Conway J.H., Sloane N.J.A., Sphere packings, lattices and groups, Grundlehren der Mathematischen Wissenschaften, Vol. 290, 3rd ed., Springer-Verlag, New York, 1999.

[8] Johnson-Freyd T., Treumann D., $H^{4}\left(\mathrm{Co}_{0} ; \mathbf{Z}\right)=\mathbf{Z} / 24$, arXiv:1707.07587.

[9] Murray M.K., Roberts D.M., Stevenson D., Vozzo R.F., Equivariant bundle gerbes, Adv. Theor. Math. Phys. 21 (2017), 921-975, arXiv:1506.07931.

[10] Noohi B., Notes on 2-groupoids, 2-groups and crossed modules, Homology Homotopy Appl. 9 (2007), 75-106, math.CT/0512106.

[11] Pressley A., Segal G., Loop groups, Oxford Mathematical Monographs, Oxford Science Publications, The Clarendon Press, Oxford University Press, New York, 1986.

[12] Schommer-Pries C.J., Central extensions of smooth 2-groups and a finite-dimensional string 2-group, Geom. Topol. 15 (2011), 609-676, arXiv:0911.2483.

[13] Tits J., Le Monstre (d'après R. Griess, B. Fischer et al.), Astérisque 121-122 (1985), 105-122.

[14] Wagemann F., Wockel C., A cocycle model for topological and Lie group cohomology, Trans. Amer. Math. Soc. 367 (2015), 1871-1909, arXiv:1110.3304.

[15] Waldorf K., Multiplicative bundle gerbes with connection, Differential Geom. Appl. 28 (2010), 313-340, arXiv:0804.4835.

[16] Waldorf K., A construction of string 2-group models using a transgression-regression technique, in Analysis, Geometry and Quantum Field Theory, Contemp. Math., Vol. 584, Amer. Math. Soc., Providence, RI, 2012, 99-115, arXiv:1201.5052.

[17] Waldorf K., Transgression to loop spaces and its inverse, II: Gerbes and fusion bundles with connection, Asian J. Math. 20 (2016), 59-115, arXiv:1004.0031. 